\title{
Medical Image of the Week: Constrictive Pericarditis
}

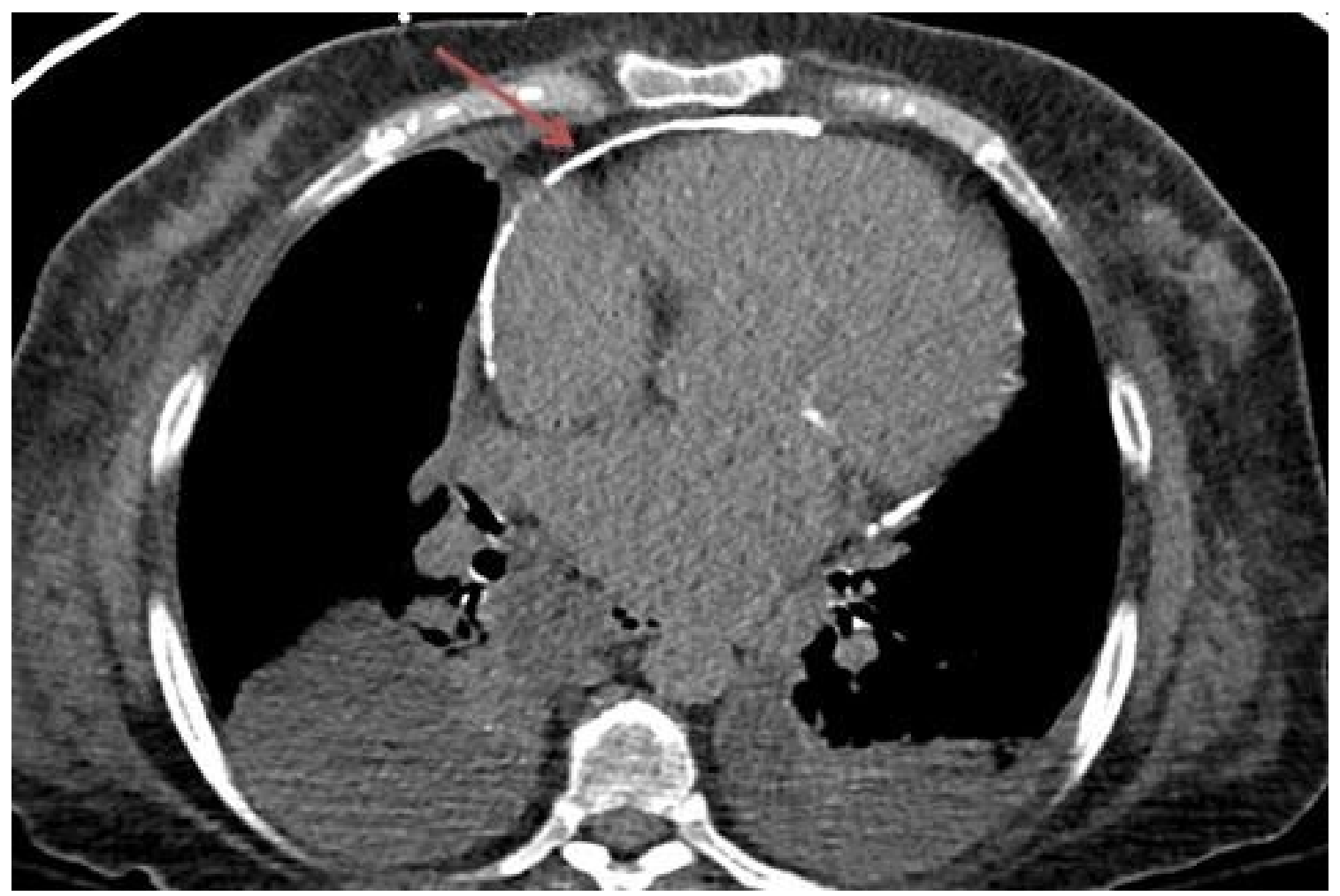

Figure 1. A computerized tomography of the chest revealed cardiomegaly, bilateral pleural effusions and pericardial calcification noted diffusely with focal regions of pericardial thickening greater than $4 \mathrm{~mm}$.

A 62-year-old woman, with a past medical history significant for oxygen dependent COPD, paroxysmal atrial fibrillation, and obstructive sleep apnea, presented to the hospital with hypoxemic respiratory failure requiring intubation and mechanical ventilation. A computerized tomography of the chest revealed cardiomegaly, bilateral pleural effusions, and pericardial calcification that was noted diffusely with focal regions of pericardial thickening greater than $4 \mathrm{~mm}$. A cardiac catheterization revealed elevated right-sided pressure; markedly elevated left ventricular end diastolic pressure; equalization of LV-RV diastolic pressures; and sharp $\mathrm{Y}$ descent on the right atrial pressure waveform; which is all suggestive of constrictive physiology. The patient was medically optimized and diuresed and eventually underwent a successful pericardiectomy.

Mohammed Alzoubaidi MD, John Bloom MD, Jarrod Mosier MD, Linda Snyder MD

Department of Pulmonary and Critical Care Medicine, University of Arizona, Tucson, AZ 